\title{
Channel Equalization for Chaos-based Communication Systems
}

\author{
Jiu-chao Feng, Chi K. Tse and Francis C.M. Lau \\ Department of Electronic and Information Engineering, Hong Kong Polytechnic University, Hong Kong, China \\ Email: fengjc@eie.polyu.edu.hk, encktse@polyu.edu.hk \& encmlau@ polyu.edu.hk
}

\begin{abstract}
The performance of chaos-based communication systems is greatly affected by non-ideal channel characteristics such as bandwidth limitation, distortion and additive noise. Systems that rely on coherent detection methods are particularly vulnerable because the process of regenerating the chaos basis signals in the detector involves a rather fragile process known as "chaos synchronisation". If channel effects can be minimized, the performance of chaos-based communication systems can be enhanced. In this paper, we study the equalization of the channel for chaotic communication systems. A channel equalizer is designed and realized by a modified recurrent neural network (RNN) for eliminating channel distortions. Results from computer simulations demonstrate the effectiveness of the equalizer as applied to a chaotic communication system.
\end{abstract}

\section{INTRODUCTION}

Research in chaos-based communications has been rapidly progressing. A number of systems have been proposed for analog and digital communications using chaotic signals as the information carrying signals. The use of chaos in communication has been catalysed by the pioneering work of Pecora and Carroll [1], who demonstrated the synchronisability of two coupled chaotic systems and hence established the possibility of achieving coherent detection in chaos-based communication. Since then, a number of conceptual approaches have been proposed for communication using chaotic signals, e.g., chaos masking, chaos-shift-keying (CSK), etc. [2]. However, the performance of such systems, especially those employing a coherent type of detection, is greatly affected by the physical channel characteristics, such as bandwidth limitation, distortion and noise [3]. In order for any chaos-based communication system to work well in practice, undesirable channel effects must be minimised so that information can be accurately recovered by the intended receiver. This paper aims to design a channel equalizer for chaos-based communication systems under practical communication environments.

Shown in Fig. 1 is the block diagram of part of a chaotic communication system, where $x$ is the transmitted signal produced by the chaotic modulator, and $h$ is the transfer function of the channel. The output of the channel, $s$, is corrupted by noise $\eta$, which is usually modeled as an additive white Gaussian (AWGN) process. At the receiver, the received signal, $y$, passes through an equalizer, which undoes the channel effects and estimates the transmitted signal. Then, the demodulator reconstructs the message.

The channel $h$ can be modeled as a linear operator. In this case the output of the channel, $s$, is simply the convolution of the input sequence $x$ with $h$, i.e., $s=h * x$. Alternatively, $h$ can be modeled as a nonlinear operator, which is given generally by $s=h(x)$ [4]. The input to the equalizer is

$$
y=s+\eta \text {. }
$$

The basic problem tackled in this paper is to reconstruct $x$ from $y$. Our method is based on the Takens embedding theory [5] imple-

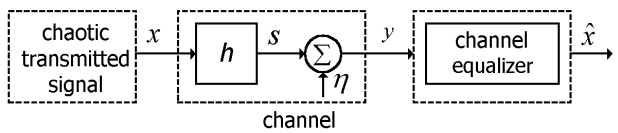

Fig. 1. Block diagram of part of a chaotic communication system showing the channel and equalizer.

mented by a recurrent neural network, as will be described in the following.

\section{Channel EQualization B y A RECURRENT-NEURAL-NETWORK CHAOS TRACKER}

Artificial neural networks (ANN's) can perform complex mapping between their input and output space. They can be used for modeling nonlinear functions and find applications in image and signal processing, but mainly for static modeling problems. For modeling dynamical systems, recurrent neural network (RNN's), by virtue of their additional output feedback, are highly suitable. Specifically, the salient property of an RNN is that the output of the hidden units are fed back at every time step to provide an additional input. This recurrence enables the filtered data of the previous period to be used as an additional input in the current period. In other words, in each time period, the network is subject to not only the new input data but also the past history of all inputs as well as their filtered versions. This additional information of the filtered input history gives a memory ability to model dynamics as well as to remove noise even when noise distribution is allowed to vary with time $[6,7]$. Such properties are highly desirable for modeling communication channels which are dynamic and may subject to varying noise distribution.

In this paper, we propose a modified RNN, as shown in Fig. 2, which is a three-layer network consisting of an input layer, a hidden layer (processing layer) and an output layer. The input vector of the network at time instant $t$ is $V(t)$, which is defined as

$$
V(t)=\left[v_{1}(t), v_{2}(t), \cdots, v_{M+N+1}(t)\right]^{T},
$$

where $v_{i}(t)(2 \leq i \leq M+1)$ is the external input which is the delayed version of $y$, i.e., $v_{i}(t)=y[t-(i-1)]$, and $v_{i}(t)(M+2 \leq$ $i \leq M+N+1$ ) is the feedback input of the $i$ th input unit at time instant $t$. Also, $N$ is the number of hidden layer units and $v_{1}$ is the bias input set as " +1 " in this paper.

The internal activity of the $i$ th hidden unit at time instant $t$ is given by

$$
r_{i}(t)=\sum_{j=1}^{M+N+1} w_{i j}(t) v_{j}(t)
$$

where $w_{i j}(t)$ is the connection weight between the $i$ th hidden unit and the $j$ th input unit at time instant $t$. At the next time step $t+1$, the output of the $i$ th neuron, $q_{i}(t+1)$, is computed by a nonlinear 


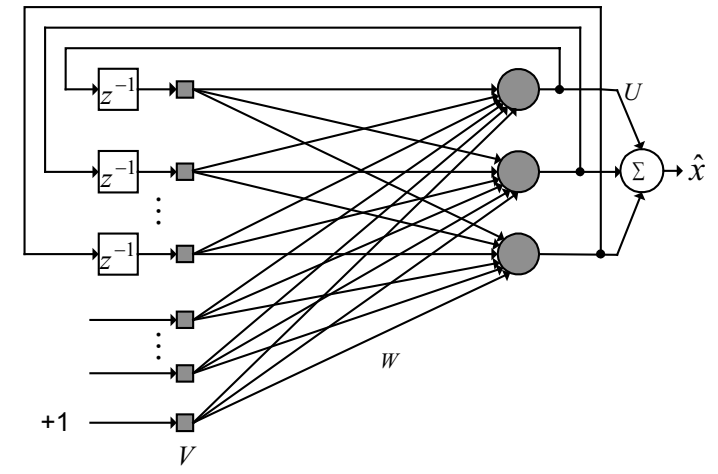

Fig. 2. Block diagram of a modified recurrent neural network.

activation function, $\varphi($.$) , yielding$

$$
q_{i}(t+1)=\varphi\left(r_{i}(t)\right) .
$$

In this study, we choose

$$
\varphi(x)=\tanh (c x),
$$

where $c$ is constant. Let $u_{i}(t)$ be the connection weight between the $i$ th hidden unit and the output unit. Then, the output of the output unit is given by

$$
\hat{x}(t+1)=\sum_{i=1}^{N}\left[u_{i}(t) q_{i}(t+1)\right] .
$$

These equations, combined with the training algorithm discussed in the following section, can be used to realize the equalization task.

\section{Training ALgORITHM}

Let $d(t+1)$ be the desired output of the output unit at time instant $t+1$. The error signal, $e(t+1)$, is

$$
e(t+1)=d(t+1)-\hat{x}(t+1) .
$$

The weight between the hidden layer and the output unit is then updated by the LMS algorithm [8], namely,

$$
u_{i}(t+1)=u_{i}(t)+\beta_{1} e(t+1) q_{i}(t+1),
$$

where $\beta_{1}$ is the learning rate. The instantaneous squared error of the network is defined as $\varepsilon^{0}(t+1)=\frac{1}{2} e^{2}(t+1)$. Also, we define the local gradient of the $i$ th hidden unit at time instant $t+1, \gamma_{i}(t+1)$, as

$$
\begin{aligned}
\gamma_{i}(t+1) & =-\frac{\partial \varepsilon^{o}(t+1)}{\partial r_{i}(t)}=-e(t+1) \frac{\partial e(t+1)}{\partial r_{i}(t)} \\
& =e(t+1) \frac{\partial \hat{x}(t+1)}{\partial r_{i}(t)}=e(t+1) u_{i}(t) \frac{\partial q_{i}(t+1)}{\partial r_{i}(t)} \\
& =e(t+1) u_{i}(t) \varphi^{\prime}\left(r_{i}(t)\right)
\end{aligned}
$$

where $\varphi^{\prime}($.$) is the derivative of \varphi$ with respect to its argument. According to the delta learning law, the weight $w_{i j}(i=$ $1,2, \cdots, N, j=1,2, \cdots, M+N+1$.) can be updated as

$$
w_{i j}^{-}(t+1)=w_{i j}(t)+\beta_{2} \gamma_{i}(t+1) v_{j}(t),
$$

where $\beta_{2}$ is the learning rate. Now define the instantaneous sum of the squared errors for the hidden layer units as

$$
\varepsilon(t)=\frac{1}{2} \sum_{k=1}^{N} e_{k}^{2}(t),
$$

where $e_{k}(t)$ is the difference (error) in the output of the $k$ th hidden unit before and after the weight $w_{i j}$ is updated. Then, the instantaneous weight is updated as

$$
w_{i j}(t+1)=w_{i j}^{-}(t+1)-\beta_{3} \frac{\partial \varepsilon(t)}{\partial w_{i j}^{-}(t+1)},
$$

where $\beta_{3}$ is the corresponding learning rate. From (4) and (11), we have

$$
\begin{aligned}
\frac{\partial \varepsilon(t)}{\partial w_{i j}^{-}(t+1)} & =\sum_{k=1}^{N} e_{k}(t) \frac{\partial e_{k}(t)}{\partial w_{i j}^{-}(t+1)} \\
& =-\sum_{k=1}^{N} e_{k}(t) \frac{\partial q_{k}^{+}(t+1)}{\partial w_{i j}^{-}(t+1)}
\end{aligned}
$$

where $q_{k}^{+}(t+1)$ is the output of the $k$ th hidden unit after the weight $w_{i j}$ is updated to $w_{i j}^{-}(t+1)$. To determine the partial derivative $\partial q_{k}^{+}(t+1) / \partial w_{i j}^{-}(t+1)$, we differentiate (3) and (4) with respect to $w_{i j}$. By applying the chain rule, we obtain

$$
\begin{aligned}
\frac{\partial q_{k}^{+}(t+1)}{\partial w_{i j}^{-}(t+1)} & =\frac{\partial q_{k}^{+}(t+1)}{\partial r_{k}^{-}(t+1)} \frac{\partial r_{k}^{-}(t+1)}{\partial w_{i j}^{-}(t+1)} \\
& =\varphi^{\prime}\left(r_{k}^{-}(t+1)\right) \frac{\partial r_{k}^{-}(t+1)}{\partial w_{i j}^{-}(t+1)}
\end{aligned}
$$

where $r_{k}^{-}(t+1)$ corresponds to the updated internal state of hidden unit $k$. By using (4), we get

$$
\begin{aligned}
\frac{\partial r_{k}^{-}(t+1)}{\partial w_{i j}^{-}(t+1)}= & \sum_{n=1}^{M+N+1} \frac{\partial}{\partial w_{i j}^{-}(t+1)}\left[w_{k n}^{-}(t+1) v_{n}(t)\right] \\
= & \sum_{n=1}^{M+N+1}\left[w_{k n}^{-}(t+1) \frac{\partial v_{n}(t)}{\partial w_{i j}^{-}(t+1)}\right. \\
& \left.+v_{n}(t) \frac{\partial w_{k n}^{-}(t+1)}{\partial w_{i j}^{-}(t+1)}\right] .
\end{aligned}
$$

Note that the derivative $\frac{\partial w_{k n}^{-}(t+1)}{\partial w_{i j}^{-}(t+1)}$ is equal to one if $k=i$ and $n=j$, and is zero otherwise. Thus, we may rewrite (15) as

$$
\frac{\partial r_{k}^{-}(t+1)}{\partial w_{i j}^{-}(t+1)}=\sum_{n=1}^{M+N+1}\left[w_{k n}^{-}(t+1) \frac{\partial v_{n}(t)}{\partial w_{i j}^{-}(t+1)}\right]+\delta_{i k} v_{j}(t)
$$

From (2), we have

$\frac{\partial v_{n}(t)}{\partial w_{i j}^{-}(t+1)}=\left\{\begin{array}{l}\frac{\partial q_{n}^{+}(t)}{\partial w_{i j}^{-}(t+1)}, \quad(n=M+2, \cdots, M+N+1) \\ 0, \quad \text { otherwise. }\end{array}\right.$

We may then combine (14), (16) and (17) to yield

$$
\begin{aligned}
\frac{\partial q_{k}^{+}(t+1)}{\partial w_{i j}^{-}(t+1)}= & \varphi^{\prime}\left(r_{k}^{-}(t+1)\right)\left[\sum_{n=M+2}^{N+M+1} w_{k n}^{-}(t+1) \frac{\partial q_{n}^{+}(t)}{\partial w_{i j}^{-}(t+1)}\right. \\
& \left.+\delta_{i k} v_{j}(n)\right],
\end{aligned}
$$

where $\delta$ is the Kronecker delta function. We may now define a dynamical system described by a triply indexed set of variables $\left\{\Omega_{i j}^{n}(t+1)\right\}$, where

$$
\Omega_{i j}^{n}(t)=\frac{\partial q_{n}^{+}(t)}{\partial w_{i j}^{-}(t+1)}
$$




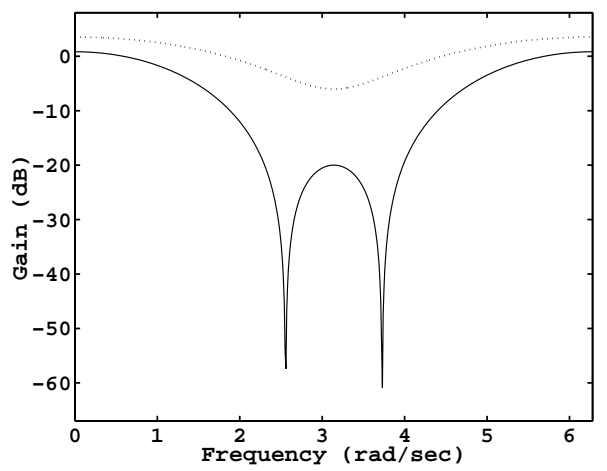

(a)

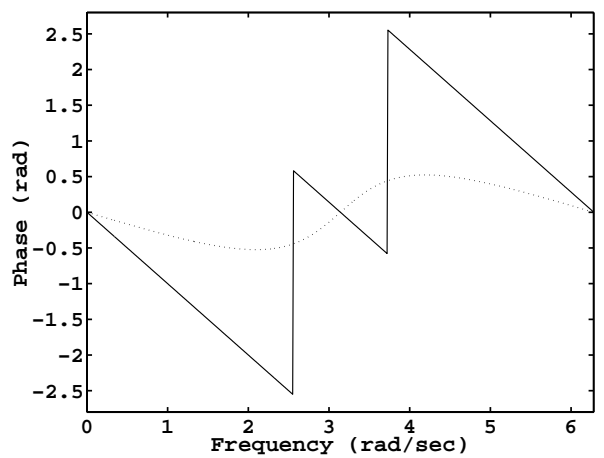

(b)

Fig. 3. Frequency responses of Channel I (dotted line) and Channel II (solid line) according to eqs. (23) and (24). (a) Magnitude responses; (b) phase responses.

where $j=1,2, \cdots, M+N+1, i=1,2, \cdots, N$, and $n=M+$ $2, \cdots, M+N+1$. For each time step and all appropriate $n, i$, and $j$, the dynamics of the system so defined is governed by

$\Omega_{i j}^{k}(t+1)=\varphi^{\prime}\left(r_{k}^{-}(t+1)\right)\left[\sum_{n=M+2}^{M+N+1} w_{k n}^{-}(t+1) \Omega_{i j}^{n}(t)+\delta_{i k} v_{j}(t)\right]$

Finally, the weight between the input layer and the hidden layer is updated by

$$
w_{i j}(t+1)=w_{i j}^{-}(t+1)+\beta_{3} \sum_{k=1}^{N} e_{k}(t) \Omega_{i j}^{k}(t+1) .
$$

The above procedure is repeatedly applied to all input sample pairs.

\section{Simulation Study}

In this section we simulate a chaotic communication system which is subject to non-ideal channel effects and additive white Gaussian noise. Our purpose is to test the performance of the proposed equalizer in combating the effects of the channel and noise.

\section{A. Chaotic Signal Transmission}

The Hénon map, as described by the following equations, will be used to evaluate the performance of the proposed equalizer.

$$
\begin{aligned}
& x_{1}(t+1)=1-\alpha_{1} x_{1}^{2}(t)+x_{2}(t) \\
& x_{2}(t+1)=\alpha_{2} x_{1}(t)
\end{aligned}
$$

where $\alpha_{1}$ and $\alpha_{1}$ are the bifurcation parameters fixed at 1.4 and 0.3 , respectively. In particular, we select $x_{2}$ as the transmitted signal.

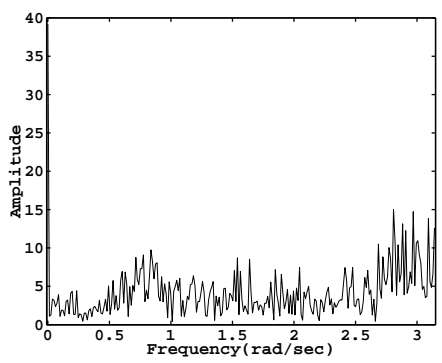

(a)

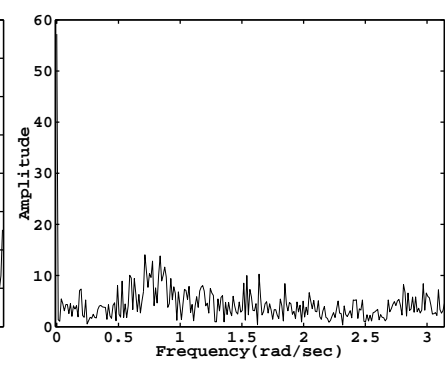

(b)
Fig. 4. FFT amplitude spectrum of signal versus frequency. (a) Transmitted signal $x_{2}$; (b) received signal $y$ after going through Channel I and being contaminated by noise at $\mathrm{SNR}=10 \mathrm{~dB}$.

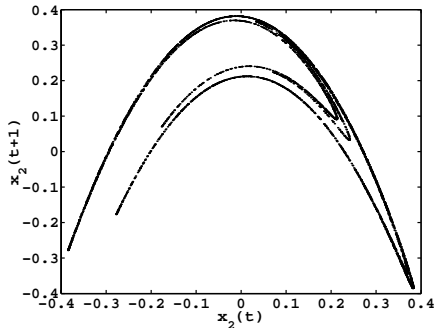

(a)

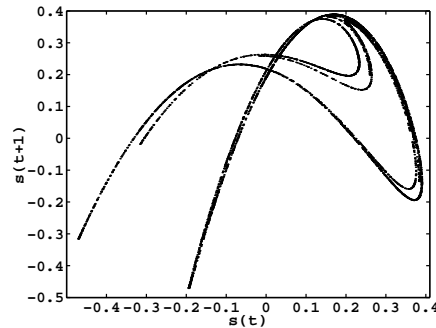

(b)

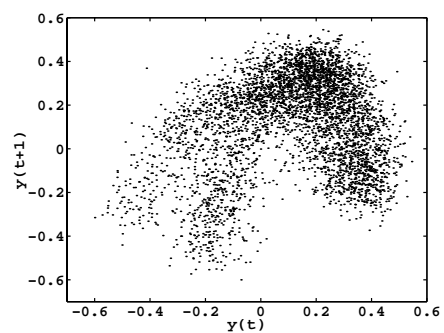

(c)

Fig. 5. Return maps reconstructed from (a) the transmitted signal $x_{2}$; (b) the distorted $s(t)$; and (c) the received signal $y$ after going through Channel I and being contaminated by noise at SNR $=10 \mathrm{~dB}$.

\section{B. Effects of Communication Channels}

In our simulation, two channel models are used to evaluate the performance of the proposed equalizer. The two channel models are described by the following $z$-domain transfer functions:

$$
\begin{aligned}
& H(z)=1+0.5 z^{-1} \text { (Channel I) } \\
& H(z)=0.3+0.5 z^{-1}+0.3 z^{-2} \text { (Channel II). }
\end{aligned}
$$

These two models are usually used to test the performance of an equalizer in digital communication systems [9]. The amplitudefrequency and phase-frequency responses of the channels are shown in Figs. 3(a) and 3(b), respectively. It is worth noting from Fig. 3(a) that the second channel has a deep spectrum null, which cannot be treated by conventional equalizers such as the linear transversal equalizer (LTE) [4].

To illustrate the channel effects, we consider Channel I. When the signal-to-noise ratio (SNR) is $10 \mathrm{~dB}$, the FFT spectra of the transmitted signal and the received signal are shown in Figs. 4(a) and 4(b). The wideband property of the transmission signal can be clearly seen from Fig. 4(a). The wideband signal goes through the channel with frequency distortion (amplitude and phase), resulting in distortion in 


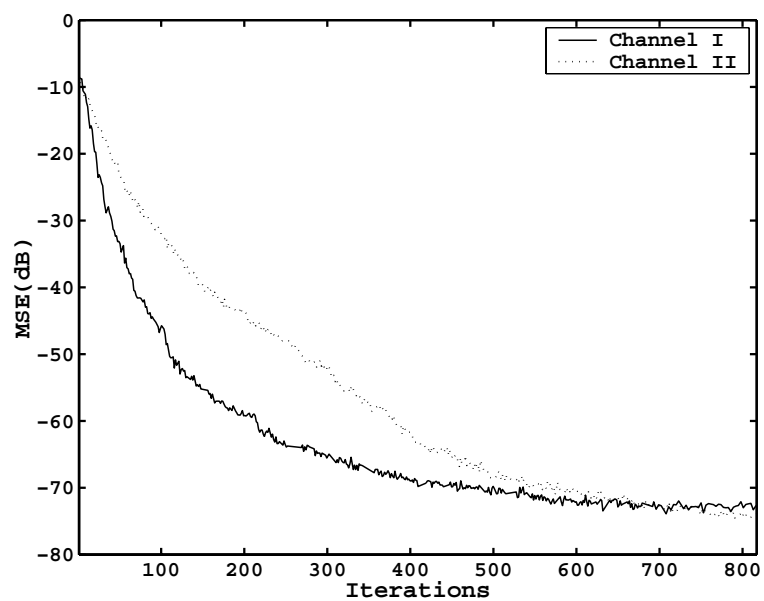

Fig. 6. MSE of the equalized samples, averaged over 40 independent realizations, versus the number of iterations.

both the amplitude and phase of the transmission signal. Also, the return maps constructed from the transmission signal, the distorted signal by the channel, and the received signal are shown in Fig. 5, in which the effects of the channel's distortion and diversity are clearly seen.

\section{Results}

The equalization for each channel model consists of two stages. The first is the training or adaptation stage where the equalizer makes use of some partially known sampling points to adapt its communication environment. When the training or adaptation is completed, actual communication begins.

In the training stage, 600 training sample sets are used, each having 7 transmitted signals and 1 known signal at the receiver. Also, noise is added to the training samples at an SNR of $10 \mathrm{~dB}$ during the training stage.

In the simulation, the RNN is assigned with $M=7$ and $N=6$. The training of the equalizer ends when mean-square-error (MSE) for all samples is less than $10^{-7}$. Figure 6 shows the MSE of the equalized training samples versus the number of iterations during training, in which the solid line is for Channel I and dotted line for Channel II. The MSE for each channel in the training stage is averaged over 40 independent realizations. It can be seen from Fig. 6 that the equalizer completes the training in 500 iterations for Channel I and 600 iterations for Channel II.

To test the performance of the trained equalizer, we evaluate the MSE of $\hat{x}_{2}$, i.e., $<\left(\hat{x}_{2}-x_{2}\right)^{2}>$, where " $<>$ " stands for the computed average. The solid line and the dotted line in Fig. 7 show the MSE versus SNR for Channel I and Channel II, respectively. When SNR is more than $14 \mathrm{~dB}$, MSE of the equalized signal is less than $-80 \mathrm{~dB}$ for the two channels. Also, it has been shown in our previous work [10] that without the equalizer the MSE will be unacceptably high even when the channel, besides the added noise, is an ideal allpass filter $(h=1)$.

\section{CONCLUSION}

This paper addresses the channel equalization problem for chaosbased communication systems. An equalizer is designed with an objective to "undo" the effects of the channel through which chaotic signals are transmitted. The design approach is based on the use of

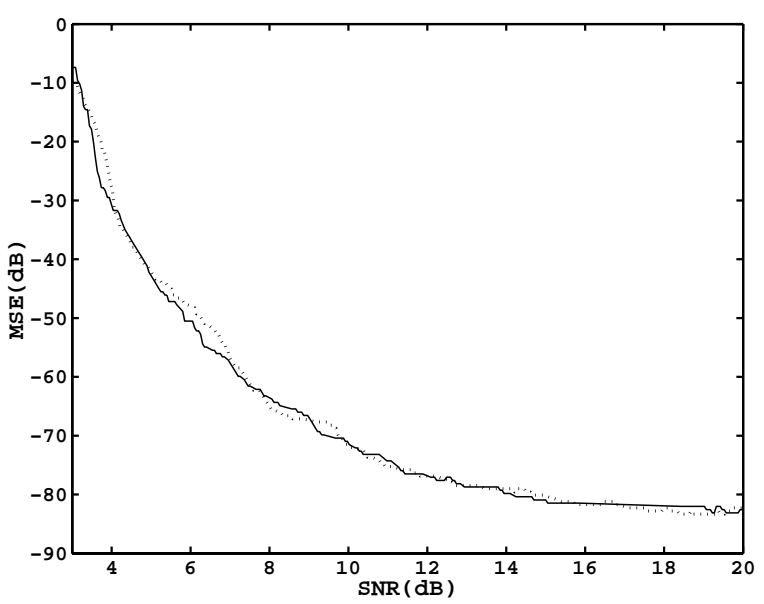

Fig. 7. MSE performance of the recovered $\hat{x}_{2}$ versus SNR, in which the solid line is for Channel I and dotted line for Channel II.

a recurrent neural network, which has the memory ability to combat dynamic channel distortion and noise, for "probing" the channel characteristics. The basic algorithm consists of a training or adaptation stage in which the channel is studied, and a message recovery stage in which actual communication takes place. Two typical channels are considered in the simulation study. It is found that the proposed recurrent-neural-network equalizer can sucessfully "undo" the channel effects for chaotic communication applications. Further work will be performed to study the equalization in the presence of time-varying noise distribution.

\section{REFERENCES}

[1] L.M. Pecora and T.L. Carroll, "Synchronization in chaotic systems", Phys. Rev. Lett., Vol. 64, pp. 821-824, 1990.

[2] M.P. Kennedy and G. Kolumbán, "Digital communication using chaos," in Controlling Chaos and Bifurcations in Engineering Systems, G. Chen (ed.), Boca Raton FL: CRC Press, 2000.

[3] G.A. Johnson, D.J. Mar, T.L. Carroll and L.M. Pecora, "Synchronization and parameter tracking in chaotic systems", Proc. of the 4th Experimental Chaotic Conference, pp. 407-412, Singapore: World Scientific, 1998.

[4] J.G. Proakis, Digital Communications, 3rd Edition, New York: McGraw-Hill, 1995.

[5] F. Takens, "Detecting strange attractors in turbulence" in $D y$ namical Systems and Turbulence, D. Rand and I. Young (ed.), pp. 366-381, Berlin: Springer-Verlag, 1981.

[6] R. Gencay and T. Liu, "Nonlinear modeling and prediction with feedforward and recurrent networks," Physica D, Vol. 108, pp. 119-134, 1997.

[7] R.J. Williams and D. Zipser, "A learning algorithm for continually running fully recurrent neural networks," Neural Computation, Vol. 1, pp. 270-280, 1989.

[8] S. Haykin, Adaptive Filter Theory, 3rd Edition, NJ: Prentice Hall, 1996.

[9] S.U.H. Qureshi, "Adaptive equalization," Proceedings of IEEE, Vol. 73, pp. 1349-1387, 1985.

[10] J-C. Feng and C.K. Tse, "On-line adaptive chaotic demodulator based on radial-basis-function neural networks," Phys. Rev. E, Vol. 63, pp. 026202:1-10, 2001. 